\section{Variable Magnification Electron \\ Holography for 2-D Mapping of Semiconductor Devices}

\author{
Y.Y. Wang, M. Gribelyuk, A. Domenicucci, J. Bruley, \\ J. Gaudiello, and M. Kawasaki* \\ IBM Microelectronic Division, ${ }^{\star} J E O L$ USA
}

\section{Introduction}

The exit electron wave from transmission electron microscopy (TEM) specimens contains both amplitude and phase information. In routine TEM imaging, only amplitude information is recorded on the recording devices (film or CCD camera) and phase information of the electron wave function normally is canceled out.

In 1947, Dennis Gabor proposed off-axis electron holography, a method of interference imaging in which the phase and amplitude components of the electron beam are obtained to correct spherical aberration of the transmission electron microscope to improve spatial resolution. In that process, the electron beam is split into the two beams: the un-scattered electron beam (i.e. the reference wave) and the image beam (or object wave) diffracted by the specimen and exiting the bottom of the specimen surface. These two waves interfere with each other to form an interference pattern, in which both amplitude and phase information can be extracted. Unfortunately, electron microscopes of the day did not produce an electron wave with sufficient coherence to yield meaningful results. With the development of highly coherent field emission electron sources, electron holography becomes an effective method to obtain information previously out of reach.

There are three critical parameters in electron holography: the width of the interference overlap, the interference fringe spacing, and the fringe contrast. These parameters determine the field of view (FOV), spatial resolution, and sensitivity of the resultant phase maps, respectively. However, in a conventional electron microscope, where the magnification of the objective lens is fixed, the FOV of electron holography is limited to either high or low magnification, shown in Fig. 1. There is a magnification gap at
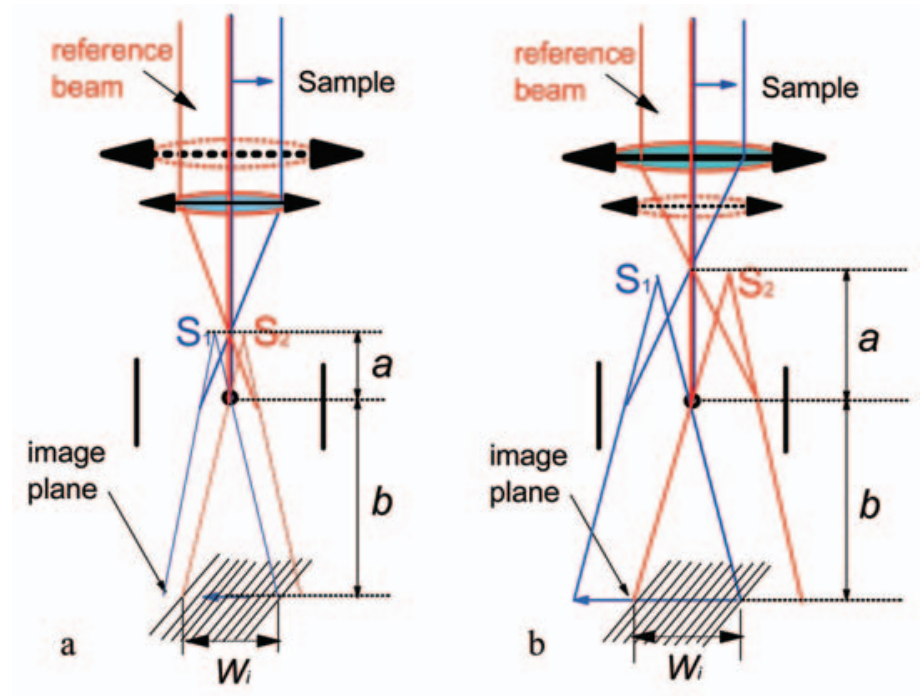

Fig.1: Electron hologram formation using a single lens in a dual lens imaging system. The interference pattern with fringe spacing, $\sigma_{i}$, and fringe width, $W_{i}$ is formed by two virtual sources, $S_{1}$ and $S_{2}$. a): low magnification mode with a lens far away from the specimen and $b$ ): high magnification mode with a lens close to the specimen.

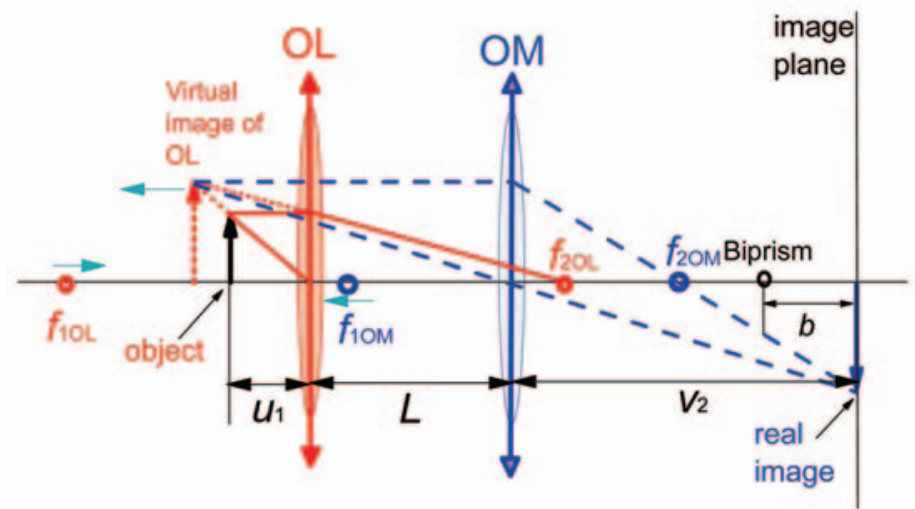

Fig.2: Ray diagram of dual lens system for electron holography. Light blue arrow indicates the movement of focal points of $O M$ and $O L$ in order to keep the final image unchanged.

intermediate magnifications in electron holography. ${ }^{1,2}$ In order to make electron holography more flexible, adjustable field of view (overlap) and spatial resolution (fringe spacing) relative to the object is desirable. To achieve this condition, the magnification of the objective lens(es) has to be changed continuously from low magnification to high magnification.

It has been shown that electron holography is particularly valuable for two-dimensional potential mapping of semiconductor devices with high spatial resolution. ${ }^{3,4}$ Such information is critical for semiconductor device development and yield improvement. The requirements of electron holography to inspect the current generation of semiconductor devices are (1) an overlap width in the range of about 100 to $1,000 \mathrm{~nm}$ for an adequate field of view (FOV), (2) fringe spacing between 0.5 and $10 \mathrm{~nm}$ for meaningful spatial resolution, (3) good visibility of fringe contrast (10-30\%) for a useful signal to noise ratio, and (4) adjustability of both the overlap and the fringe spacing relative to the specimen for flexibility.

Based on these requirements, a method of using the two objective lenses on a JEOL $2010 \mathrm{~F}$ simultaneously to provide a continuously varying magnification of the specimen at the intermediate imaging plane was developed. This dual lens operation allows electron holography to be performed from low to high magnification and provides the FOV and fringe spacing necessary for two-dimensional (2D) junction mapping of semiconductor devices. ${ }^{5}$ Although, this method was developed for semiconductor device characterization, its usefulness is not limited to that application.

\section{Formation of off-axis TEM electron holography}

A coherent, plane electron wave, such as that formed from a field emission tip, is necessary for electron holography. As shown in Fig.1, the object is inserted in the object plane so that half of an incident wave passes without scattering. The other half is scattered by the specimen to form an electron wave $\Psi(\mathrm{r})=A(\mathrm{r}) \exp [\mathrm{i} \phi(\mathrm{r})]$ at the object exit plane, where $A(\mathrm{r})$ and $\phi(\mathrm{r})$ are the amplitude and the phase of the complex electron wavefunction. To form electron holograms, a positive voltage is applied to the biprism filament, while the electrodes are grounded, which causes one beam to split into two beams interfering with each other. As illustrated in Fig.1, the off-axis electron hologram interference pattern can be thought of as being formed from two virtual sources, $S_{1}$ and $S_{2}$, of finite size $(\delta)$ positioned above the biprism and separated by a distance $d$. The interference pattern $I(\mathrm{r})$, i.e. the electron hologram, consists of fringes, in which amplitude and position are modulated by the 


\section{for Specimen Preparation.}

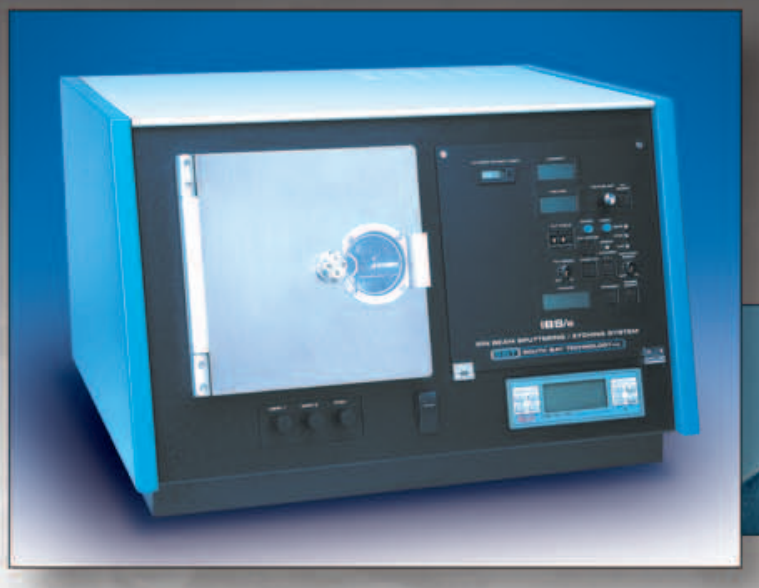

Ion Beam Sputter Deposition and Etching System

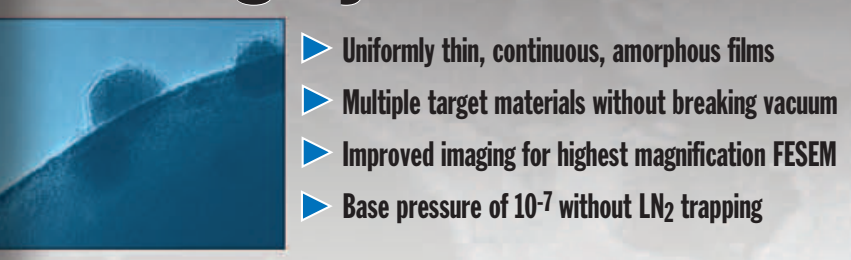

\section{Plasma Cleaner}

Removes hydrocarbons without damaging specimen Intuitive front panel controls $<$ Ideal for multi-user environments 4 Large chamber accommodates samples 4 up to $3^{\prime \prime}$ tall and 6" diameter
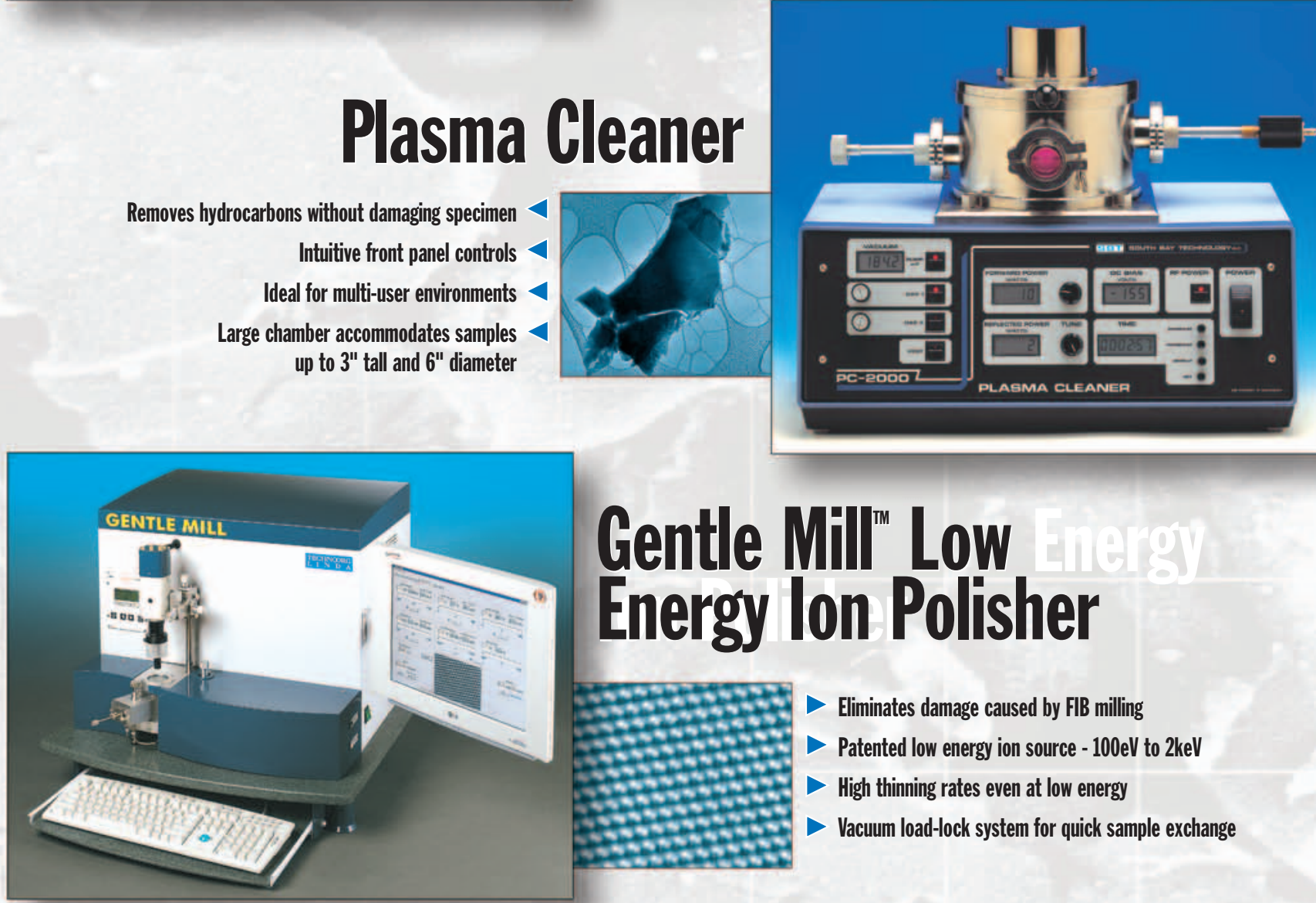

\section{Gentle Mill" Low} Energy lon Polisher

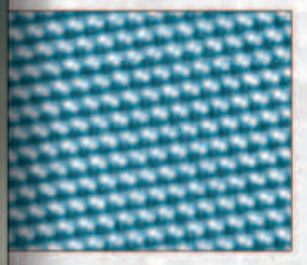

- Eliminates damage caused by FIB milling

Patented low energy ion source - $100 \mathrm{eV}$ to $2 \mathrm{keV}$

High thinning rates even at low energy

V Vacuum load-lock system for quick sample exchange

www.southbaytech.com

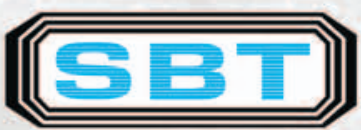

SOUTH BAY TECHNOLOEY, INE.

1120 Via Callejon | San Clemente, CA 92673 | U.S.A. | 949-492-2600 | Fax: 949-492-1499

Toll Free: 800-728-2233 | email: info@southbaytech.com | www.southbaytech.com 
scattered electron wave $\Psi(\mathrm{r})$ :

$I(r)=1+A^{2}(r)+2 \mu A(r) \cos \left(2 \pi q_{c} r+\varphi(r)+\theta\right)$

where $q_{c}$ denotes the carrier frequency of the interference fringes (related to spatial resolution), $\mu$ is the fringe contrast, and $\theta$ is the constant phase shift.

The phase map, which is related to mean inner potential, and the amplitude map, which is related to specimen thickness, can be extracted through a fast fourier transformation (FFT) of the hologram and an inverse FFT of an appropriately sized side band. Mean inner potential, which is related to junction profile, can be calculated through these two maps. ${ }^{6}$

As discussed above, in order to produce meaningful electron holograms, proper fringe width and fringe spacing relative to the region of interest are required. The fringe spacing, $\sigma_{o b j}$, and fringe width, $W_{o b j}$, relative to the object, were described by Lichte as: $:^{7,8}$

$$
\sigma_{o b j}=\frac{1}{M_{o}} \frac{\lambda}{2 \gamma_{o} V_{b}}\left(1+\frac{b}{a}\right)
$$

and

$$
W_{o b j}=\frac{1}{M_{o}}\left[2 \gamma_{o} V_{b} b-2 r_{b}\left(1+\frac{b}{a}\right)\right]
$$

where $a$ is the distance between the virtual sources and biprism position, $b$ is the distance between the image plane and the the biprism position, $\lambda$ is the wavelength of the electron beam, and $V_{b}$ is the biprism voltage, $\gamma_{0}$ is a constant related to microscope setup, and $r_{b}$ is the radius of biprism wire. ${ }^{8,9} M_{0}$ is the magnification of the imaging lens(es). The above equations show that the magnification is a major factor that determines the fringe spacing and width relative to the object and hence, a major factor in determining the FOV and spatial resolution of the hologram.

Fringe contrast is another important parameter in electron holography and can be written as: ${ }^{5}$

$$
\mu=\frac{I_{\max }-I_{\min }}{I_{\max }+I_{\min }}=\frac{\sin (\beta)}{\beta}
$$

where

$$
\beta=\frac{2 \pi \delta}{\lambda} \frac{\gamma_{o} b}{a+b} V_{b}
$$

The fringe contrast, along with the electron dose determines the minimum detectable voltage in the potential maps derived from electron holograms. Based on Eqs. (2) and (3), larger biprism voltages lead to larger fringe widths and smaller fringe spacings, both preferred for most analyses. However, fringe contrast decreases with increased biprism voltage due to the finite size of the electron source, as indicated by Eqs. (4) and (5). The control of fringe width, spacing, and contrast by varying the biprism voltage alone, limits the operational range of a TEM set up for electron holography. By continuously altering the magnification of the imaging lenses, (see Eqs.(2) and (3)), the fringe width $\left(W_{\mathrm{obj}}\right)$ and spacing $\left(\sigma_{\mathrm{obj}}\right)$ can be changed without significantly degrading fringe contrast, widening the operational range for electron holography. In order to achieve this, a dual lens operational mode was implemented to adjust the magnification of the imaging system. ${ }^{5}$
Optical ray diagram and experimental results for the dual

\section{lens method}

Figure 2 illustrates the optical diagram for dual lens operation mode. The voltage of the objective lens $(\mathrm{OL})$ is set so that the position of the object is behind the first focal point $\mathrm{F}_{1 \mathrm{OL}}$, but before the lens, thereby forming a virtual image of the object. The second objective lens $(\mathrm{OM})$ is used to project a real image of the OL virtual image onto the intermediate image plane beyond the biprism. As shown in Fig.2, when the focal point of OL gets closer to the specimen, the virtual image moves further away from the specimen and is magnified. In order to refocus the virtual image onto the same image plane, the focal length of $\mathrm{OM}$ is increased (strength of $\mathrm{OM}$ decreases) to compensate for the movement of the virtual image position, as indicated in the figure by blue arrows. So the magnification of the specimen at the image plane, $M_{\mathrm{obj}}$, can be adjusted by varying the focal length of the first objective lens (OL) to give a virtual image of variable size which is refocused to the intermediate image plane by the objective minilens (OM).

Fig.3 summarizes experimental results for fringe width and fringe spacing relative to OL lens excitation. The fringe spacing and width relative to the object are plotted against OL excitation at a constant biprism voltage (20V). The fringe spacing and width decreases as OL increases, but at different rates. In addition, fringe spacing levels-off for OL voltages above 3.0V. The behavior of fringe width with OL can be explained by the increasing of the magnification of the imaging system as OL strength increases. According to equation (3), if we ignore the second term in the equation and assume that the imaging plane is fixed, the fringe width relative to the specimen is inversely proportional to the magnification of the imaging lens. The relationship between fringe spacing and OL strength depends on the virtual source position in addition to the

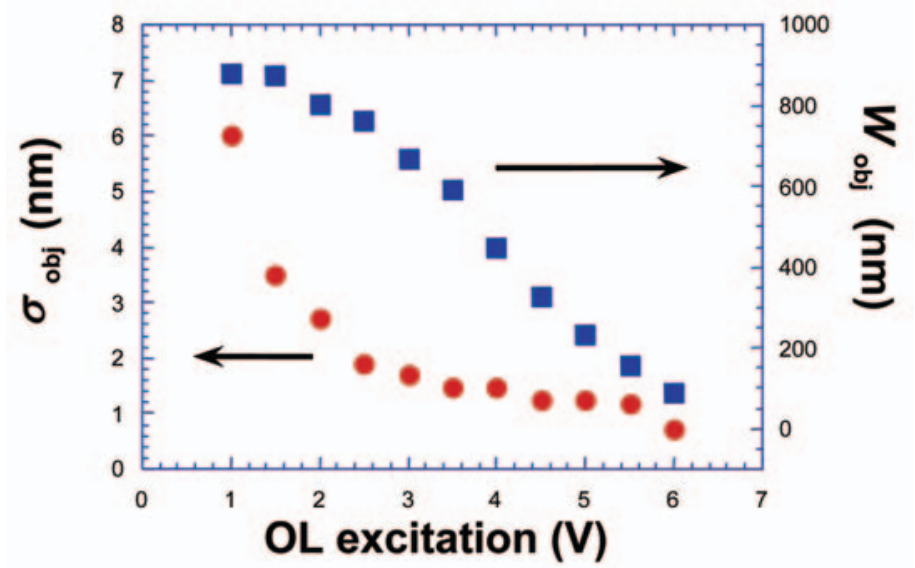

Fig.3: Fringe spacing, $\sigma_{o b j}$ (circle), and fringe width, $W_{o b j}$ (square), relative to the object are plotted against $O L$ excitation with a constant biprism voltage of $20 \mathrm{~V}$.

magnification of the imaging system as shown in equation (2). A detailed discussion concerning this can be found in reference 5 .

The graphical results show that the range of values for field of view and fringe spacing necessary for semiconductor characterization are achieved. Moreover, although the fringe contrast is strongly dependent on the biprism voltage, it is only weakly dependent on the OL excitation. In all, the data show that the dual lens mode leads to a large operational space for electron holography without sacrificing fringe contrast, which determines the signal to noise ratio in the amplitude and phase (potential) maps derived from the electron holograms. 

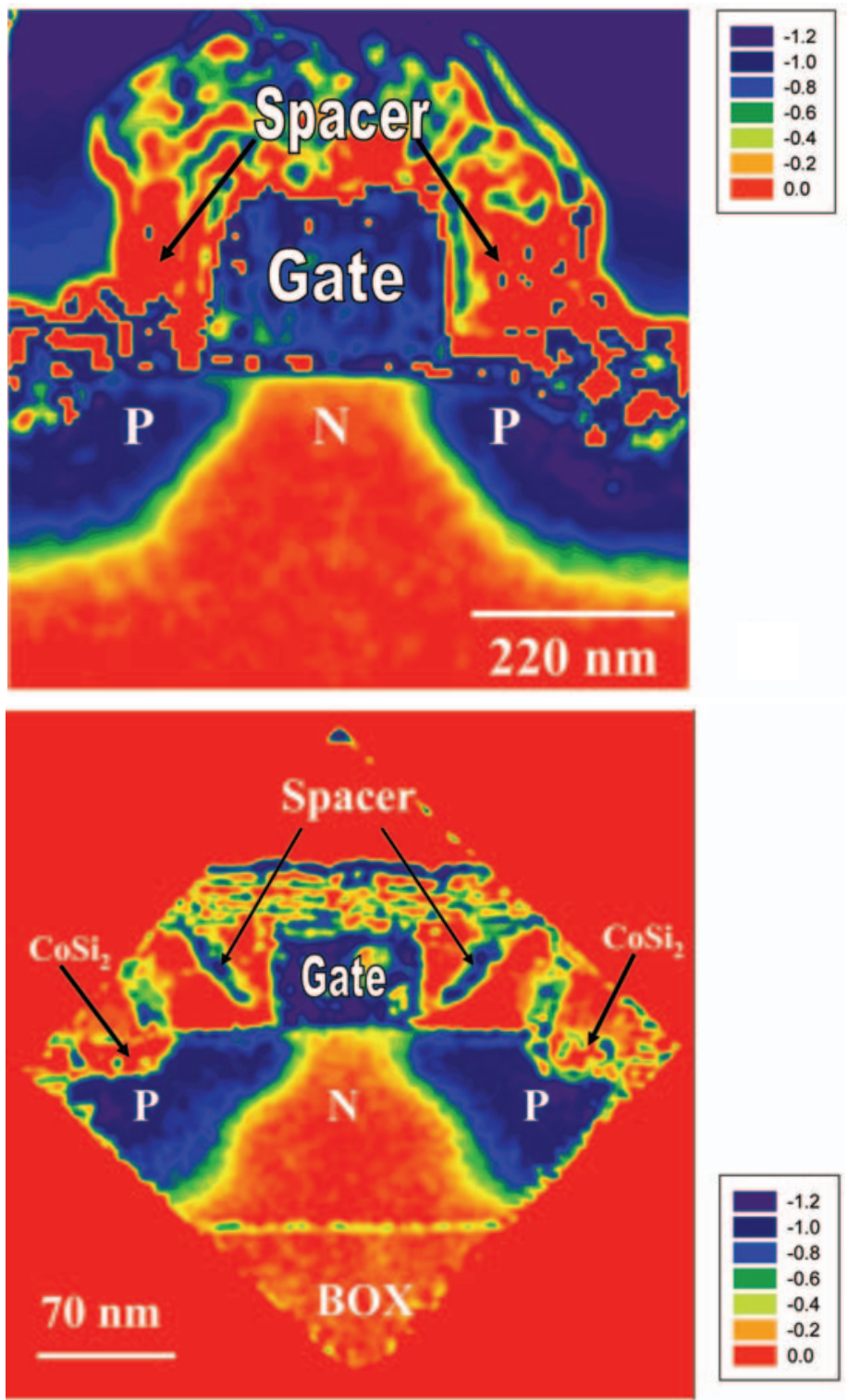

Fig.4: a) and b) are potential maps of pFET devices made with the dual lens method and different spatial resolution. The experimental conditions are shown in table I.

\section{Example}

To demonstrate the flexibility of the dual lens method, electron holograms of $p$ FET devices with poly silicon gate lengths of $220 \mathrm{~nm}$ and $70 \mathrm{~nm}$ on bulk Si and on silicon on insulator (SOI) substrates were obtained, respectively. We also analyzed the $p$-junction in a $n$-well for a narrow Si device structure by electron holography.

Specimens were prepared by mechanically polishing to an initial thickness of $\sim 400 \mathrm{~nm}$ and using ion milling to reduce the thickness to a final value of $\sim 200-300 \mathrm{~nm}$. The specimens were then coated on one side with $\sim 20 \mathrm{~nm}$ of carbon to reduce the effects of charging. Two holograms were obtained using a biprism voltage of $20 \mathrm{~V}$ : the first one is the hologram with the specimen and the second one is the hologram without the specimen, known as a reference hologram. Phase and amplitude maps were obtained from these two holograms through Fast Fourier and inverse Fast Fourier Transformations (FFT). Potential and amplitude maps were extracted from the phase map via the method described in reference 6 .

Contour potential maps for the two devices are shown in Fig.4. Both devices exhibit symmetric doping profiles and the expected $1.0 \mathrm{~V}$ potential drop across the junctions. The intensity of the maps near the sides of the gates (side wall spacers and $\mathrm{CoSi}_{2}$ ) changes sign abruptly due to phase wrapping. As summarized in Table I, both $\sigma_{o b j}$ and $W_{o b j}$ can be scaled by simply varying OL excitation without losing fringe contrast and, ultimately, the signal to noise ratio of the maps. This allows the characterization of different size devices with the same signal sensitivity, which is required for electron holography to be a practical method to investigate the physical properties of not only semiconductor devices, but also other materials.

In high performance CMOS logic devices, low dose ion implants are placed between the channel and the low resistance (high dose) source/drain regions. In Fig. $4 \mathrm{~b}$, the upper curved portion of the P$\mathrm{N}$ junction on either side of the device channel represents the result of such a low dose implant, which is clearly visible due to the high spatial resolution in the hologram from which the potential map was reconstructed.

Another example illustrating the usefulness of electron holography
TEM Bright Field Image

a

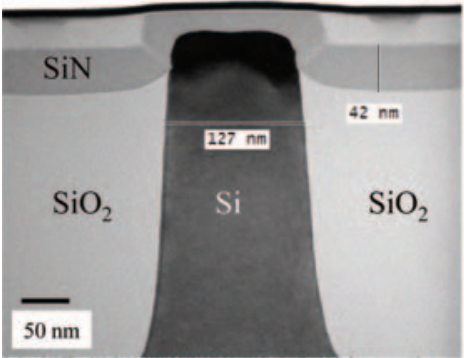

Potential map

\section{b}

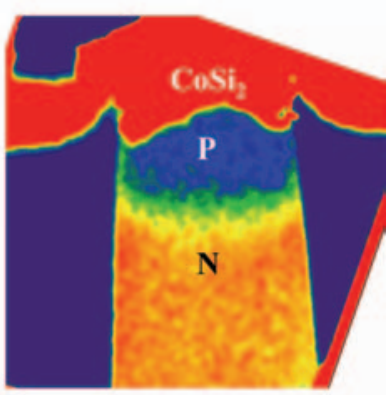

Fig.5: P-diffusion to N-well profile for narrow Si. At the edge of Si near $\mathrm{SiO}_{2}$ wall, the junction is slightly tilted towards $\mathrm{CoSi}_{2}$.

is the junction mapping of a narrow Si region containing an electrical junction (shown in Fig.5a). In typical device layouts, this is usually the contact region or the region that makes connection between one device and another. Sometimes, a high leakage current is observed from the contact region to the substrate in this narrow $\mathrm{Si}$ diffusion region. In order to understand the leakage path, a junction map was acquired by electron holography, (Fig.5b). A P-N junction is clearly shown in the potential map. Near the edge of the Si diffusion region, the junction is slightly curved upwards towards the $\mathrm{CoSi}_{2}$. The slight displacement of the junction near the edge of the insulator could be due to either implant shadowing or $\mathrm{B}$ loss at the $\mathrm{Si}$ to $\mathrm{SiO}_{2}$ interface. Without high spatial resolution, it would be difficult to detect this small shift of the junction near the edge of Si diffusion region.

\section{Conclusion}

Dual lens electron holography with a wide range of field of view and spatial resolution for semiconductor junction characterization is reported in this paper. The method has the advantage of allowing the fringe width and fringe spacing in electron holograms to be changed independently from the fringe contrast. This results in better spatial resolution and voltage sensitivity for the characterization of semiconductor devices using 2-D potential mapping. To demonstrate the utility of the method, three potential maps with different spatial resolution were reported in this paper.

\section{Acknowledgment}

We would like to acknowledge the very helpful assistance and discussions provided by Dr. Y. Zhu and Dr. M. Schofield at Brookhaven National Lab during the preliminary investigation of the subject on a JEOL 3000F. We would also like to thank Dr. L. Gignac at IBM Watson Research Lab for fruitful discussions and assistance in the preliminary 
investigations. Mr. S. Klepeis and Mr. L. Kimball of IBM are acknowledged for their skillful work in preparing the TEM specimens used in this study.

\section{Table I.}

Experimental conditions of the two potential maps shown in Figs. $4 \mathrm{a}$ and $4 \mathrm{~b}$

\begin{tabular}{|l|c|c|}
\hline & $\begin{array}{c}220 \mathrm{~nm} \text { Gate } \\
(\text { Fig.4a) }\end{array}$ & $\begin{array}{c}70 \mathrm{~nm} \text { Gate } \\
\text { (Fig.4b) }\end{array}$ \\
\hline OL voltage $(\mathrm{V})$ & 2.0 & 3.5 \\
\hline Fringe Spacing, $\sigma_{\text {obj }}(\mathrm{nm})$ & 2.5 & 1.4 \\
\hline Fringe Width, $W_{\text {obj }}(\mathrm{nm})$ & 800 & 575 \\
\hline$M_{\text {obj }}$ & 5.7 & 14.8 \\
\hline Contrast $($ reference hologram) & $10 \%$ & $11 \%$ \\
\hline
\end{tabular}

\section{References}

1. R.E. Dunin-Borkoski, unpublished results

2. M.A. Schofield, Y. Zhu, L.Wu, V.V. Volkov, and M. Malac, JEOL news-electron optics instrumentation, Vol. 36E (1), (July 10, 2001) P.2

W.D. Rau, P. Schwander, R.H. Baumann, F.H. W. Hoppner, A. Ourmazd, Physical Review Letter, 82, (1999), 2614

M.A. Gribelyuk, M. R. McCartney, J. Li, C.S. Murthy, P. Ronsheim, B. Boris, J. S. McMurray, S. Hedge, and D.J. Smith, Physical Review Letters, 89, (2002)

Y.Y. Wang, M. Kawaski, J. Bruley, M. Gribelyuk, A. Domenicucci, J. Gaudiello, to be published in Ultramicroscopy; Y.Y. Wang, M. Kawaski, J. Bruley, A. Domenicucci, M. Gribelyuk, and J. Gaudiello, IBM and JEOL USA patent application, submitted to US patent office; Y.Y. Wang, M. Kawaski, J. Bruley, M. Gribelyuk, A. Domenicucci, J. Gaudiello, JEOL news, 39, (2004), 6 .

M.R. Mccartney, Ultramicroscopy, 53, (1994), 283.

H. Lichte, Ultramicroscopy 64 (1996), 79-86.

W.D. Rau and H. Lichte, Introduction to Electron Holography Edited by E. Volk, L. Allard, and D.C. Joy, 1999 Kluwer Academic/Plenum Publishers, New York, p.208

H. Lichte, Advances in Optical and Electron Microscopy, Vol.12, p. 25, Academic Press, 1991.

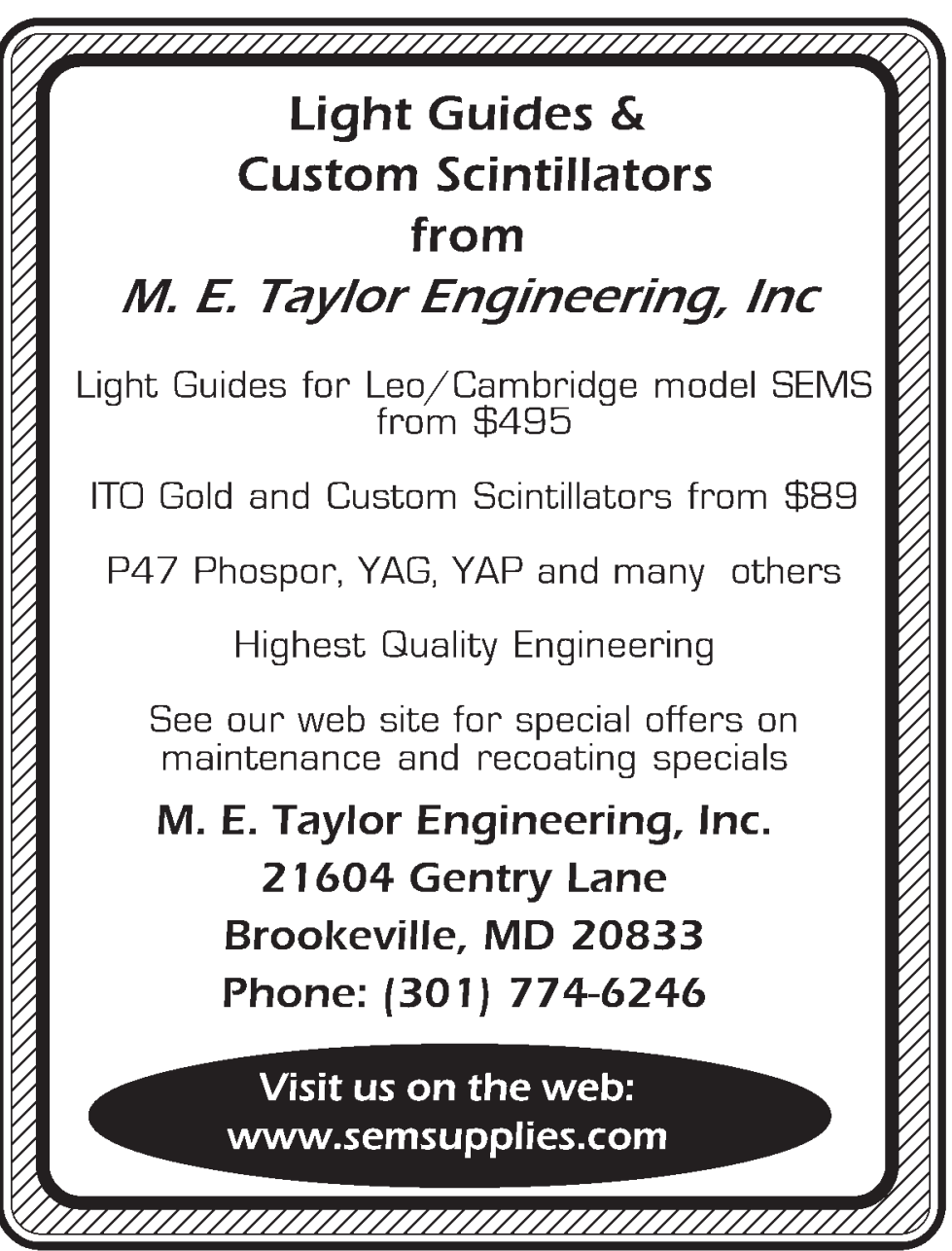

Quality Preparation

\section{Quality Results}

Advanced EM Specimen Preparation Instiments
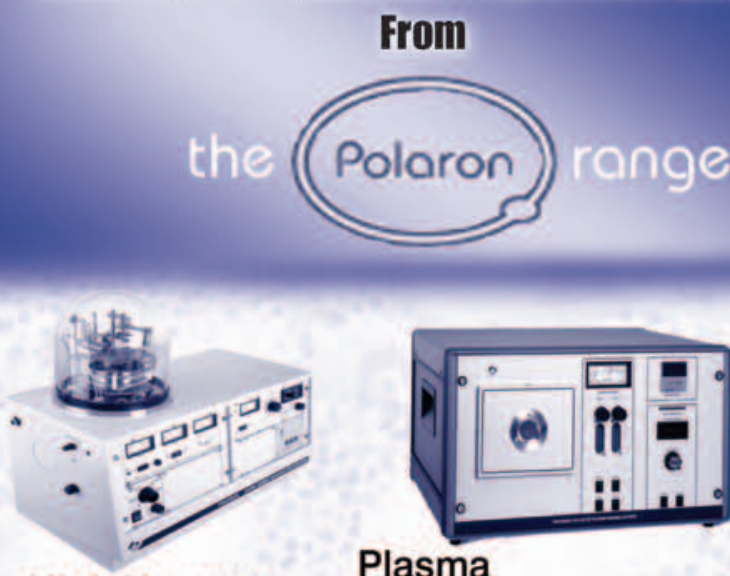

High Vacuum Evaporation

\section{Plasma}

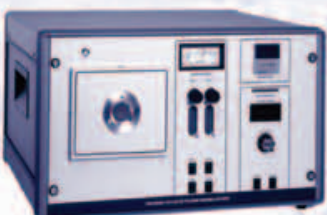

Etching/ashing

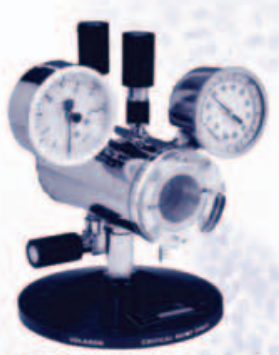

Critical Point Drying

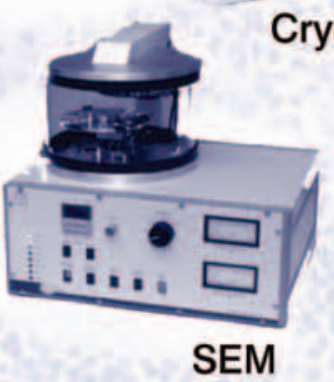

Coating

\section{Introducing the}

\section{E6500 Evaporator}

\section{Low Cost - Full Features - Revolutionary Design}

\section{EூRS}

ADDING BRIIIIANCE TO YOUR VISION

800-992-9037 or 413-786-9322

email:ebs@ebsciences.com

www.ebsciences.com 